\title{
Two-Dimensional Cubic Convolution
}

\author{
Stephen E. Reichenbach, Member, IEEE, and Frank Geng, Member, IEEE
}

\begin{abstract}
This paper develops two-dimensional (2-D), nonseparable, piecewise cubic convolution (PCC) for image interpolation. Traditionally, PCC has been implemented based on a onedimensional (1-D) derivation with a separable generalization to two dimensions. However, typical scenes and imaging systems are not separable, so the traditional approach is suboptimal. We develop a closed-form derivation for a two-parameter, 2-D PCC kernel with support $[-2,2] \times[-2,2]$ that is constrained for continuity, smoothness, symmetry, and flat-field response. Our analyses using several image models, including Markov random fields, demonstrate that the 2-D PCC yields small improvements in interpolation fidelity over the traditional, separable approach. The constraints on the derivation can be relaxed to provide greater flexibility and performance.
\end{abstract}

Index Terms-Cubic convolution, image reconstruction, image/video processing, interpolation and spatial transformations.

\section{INTRODUCTION}

$\mathbf{I}$ MAGE interpolation is the process of defining a spatially continuous image from a set of discrete samples. It is fundamental to many digital image processing operations, such as translation, scaling, rotation, and geometric correction. These general operations require image values at locations for which no sample is available. Typically, the interpolated values at these locations are computed as a weighted average (or convolution) of the neighboring image samples. The weighting function used in local convolution is called the kernel. Common kernels for image reconstruction include nearest neighbor, bilinear, and piecewise cubic.

Piecewise cubic convolution (PCC) has been used for image interpolation since the 1970's [1]. The traditional PCC kernel has been defined as the separable generalization of a symmetric, one-dimensional (1-D) function consisting of cubic polynomial pieces between knots at $\{-2,-1,0,1,2\}$. Parametric cubic convolution is a popular approach that imposes constraints to insure continuity and smoothness leaving one parameter that can be used to tune the kernel for the image [2]. Because PCC provides a good compromise between computational complexity and interpolation accuracy, it is used widely in remote sensing [3] and other applications. However, typical scenes and imaging systems are not separable and so the separable derivation is suboptimal.

Manuscript received December 5, 2000; revised February 12, 2003. This work was supported by the National Aeronautics and Space Administration, the Nebraska NASA Space Grant Consortium, and the National Science Foundation Digital Government Program. The associate editor coordinating the review of this manuscript and approving it for publication was Prof. Yucel Altunbasak.

The authors are with the Computer Science and Engineering Department University of Nebraska-Lincoln, Lincoln NE 68588-0115 USA (e-mail reich@unl.edu).

Digital Object Identifier 10.1109/TIP.2003.814248
In this paper, we develop a two-dimensional (2-D), symmetric, PCC kernel defined on $[-2,2] \times[-2,2]$. The general 2-D piecewise polynomial of degree six is constrained to yield a symmetric cubic kernel that ensures smooth, continuous interpolation. Theoretical analyses and example results both show that this 2-D convolution kernel yields slightly better interpolation than the traditional separable PCC kernel. Section II formulates the 2-D, nonseparable convolution kernel subject to constraints for symmetry, continuity, smoothness, and flat-field response constraints. Section III describes mathematical analyses to determine the best values for the two parameters in the convolution kernel. Section IV presents an analysis of performance. Section V illustrates an example image. Section VI summarizes this work and suggests future directions.

\section{Two-DIMENSIONAL DERIVATION}

\section{A. Traditional Separable Derivation}

It is useful to review the traditional 1-D derivation of the separable kernel in order to introduce both concepts and notation. One-dimensional, PCC interpolation is implemented by convolving the samples of a digital image $p$ with a piecewise-cubic kernel $f$ to define the continuous result $r$

$$
r(x)=\sum_{m=-\infty}^{\infty} p[m] f(x-m) \quad-\infty<x<\infty .
$$

For notational convenience, the spatial coordinates are normalized in units of the sampling interval.

A symmetric kernel is defined piecewise by cubic polynomials in the intervals $|x| \leq 1$ and $1<|x| \leq 2$. For $|x|>2$, the kernel is zero. In its most general (symmetric) form, there are eight degrees of freedom

$$
f(x)= \begin{cases}a_{3}|x|^{3}+a_{2}|x|^{2}+a_{1}|x|+a_{0} & \text { if }|x| \leq 1 \\ b_{3}|x|^{3}+b_{2}|x|^{2}+b_{1}|x|+b_{0} & \text { if } 1<|x| \leq 2 \\ 0 & \text { otherwise }\end{cases}
$$

To insure continuous, smooth interpolation and flat-field response, it is necessary to impose constraints at the knots. A smooth function is continuous

$$
\begin{aligned}
\lim _{x \rightarrow 1-} f(x) & =\lim _{x \rightarrow+} f(x) \\
f(2) & =0
\end{aligned}
$$

and has a continuous first-derivative

$$
\begin{aligned}
f^{\prime}(0) & =0 \\
\lim _{x \rightarrow 1-} f^{\prime}(x) & =\lim _{x \rightarrow 1+} f^{\prime}(x) \\
f^{\prime}(2) & =0 .
\end{aligned}
$$


A flat-field response means that if the digital image has constant pixel values, then the interpolated image will have constant value. For unity response, this requires that for any $x$ :

$$
\forall x, \quad \sum_{m=-\infty}^{\infty} f(x-m)=1 .
$$

Interpolation requires that the function value be 0 for integer abscissa except at the origin

$$
f(1)=0 .
$$

The seven constraints in (3)-(9) leave one degree of freedom which can be identified with the slope of the kernel at $x=1$. The resulting 1-D PCC kernel is

$$
f(x)= \begin{cases}(\alpha+2)|x|^{3}-(\alpha+3)|x|^{2}+1 & \text { if }|x| \leq 1 \\ \alpha|x|^{3}-5 \alpha|x|^{2}+8 \alpha|x|-4 \alpha & \text { if } 1<|x| \leq 2 \\ 0 & \text { otherwise }\end{cases}
$$

where $\alpha$ is the first derivative or slope of the kernel at $x=1$. The kernel function can be written as the sum of a component independent of $\alpha$ and a component weighted by $\alpha$

$$
f(x)=f_{0}(x)+\alpha f_{1}(x)
$$

where

$$
\begin{aligned}
& f_{0}(x)= \begin{cases}2|x|^{3}-3|x|^{2}+1 & \text { if }|x| \leq 1 \\
0 & \text { otherwise }\end{cases} \\
& f_{1}(x)= \begin{cases}|x|^{3}-|x|^{2} & \text { if }|x| \leq 1 \\
|x|^{3}-5|x|^{2}+8|x|-4 & \text { if } 1<|x| \leq 2 \\
0 & \text { otherwise. }\end{cases}
\end{aligned}
$$

The slope $\alpha=-1$ has been used and matches the slope of the sinc function ${ }^{1}$ at $x=1$ [1]. The slope $\alpha=-(1 / 2)$ offers third-order convergence [4] and guaranteed superiority to nearest-neighbor (first-order convergence) and linear interpolation (second-order convergence) with respect to sampling-andinterpolation error for sufficiently sampled scenes. Interestingly, 1-D PCC with $\alpha=-1$ does not provide that guarantee [2]. Finally, if the autocorrelation (or power-spectrum) of the image is known, the slope can be set to optimize expected performance [2].

The Fourier transforms of the kernel function components in (12) and (13) are

$$
\begin{aligned}
& \hat{f}_{0}(u)=\frac{3}{(\pi u)^{2}}\left(\operatorname{sinc}^{2}(u)-\operatorname{sinc}(2 u)\right) \\
& \hat{f}_{1}(u)=\frac{2}{(\pi u)^{2}}\left(3 \operatorname{sinc}^{2}(2 u)-2 \operatorname{sinc}(2 u)-\operatorname{sinc}(4 u)\right) .
\end{aligned}
$$

The 2-D separable generalization of the 1-D PCC kernel is

$$
\begin{aligned}
f_{s}(x, y)= & f(x) f(y) \\
= & \left(f_{0}(x)+\alpha f_{1}(x)\right)\left(f_{0}(y)+\alpha f_{1}(y)\right) \\
= & f_{0}(x) f_{0}(y)+\alpha\left(f_{0}(x) f_{1}(y)+f_{1}(x) f_{0}(y)\right) \\
& +\alpha^{2} f_{1}(x) f_{1}(y) .
\end{aligned}
$$

${ }^{1}$ The sinc function is the interpolation function (or point-spread function) of the ideal low-pass filter for images sampled at the Nyquist rate or higher

$$
\operatorname{sinc}(u)= \begin{cases}(\sin (\pi u)) /(\pi u), & \text { if } u \neq 0 \\ 1, & \text { if } u=0\end{cases}
$$

\section{B. Nonseparable Derivation}

The general, 2-D, symmetric, piecewise polynomial with degree six on the interval $[-2,2] \times[-2,2]$ is defined by the function in the first quadrant as

$$
f(x, y)=\left\{\begin{array}{ll}
f_{a}(x, y)=\sum_{j=0}^{6} \sum_{k=0}^{6-j} a_{j k} x^{j} y^{k} & 0 \leq x \leq 1,0 \leq y \leq 1 \\
f_{b}(x, y)=\sum_{j=0}^{6} \sum_{k=0}^{6-j} b_{j k} x^{j} y^{k} & 1<x \leq 2,0 \leq y \leq 1 \\
f_{c}(x, y)=\sum_{j=0}^{6} \sum_{k=0}^{6-j} c_{j k} x^{j} y^{k} & 1<x \leq 2,1<y \leq 2 \\
f_{d}(x, y)=\sum_{j=0}^{6} \sum_{k=0}^{6-j} d_{j k} x^{j} y^{k} & 0 \leq x \leq 1,1<y \leq 2
\end{array} .\right.
$$

This general form has $112=28 \times 4$ parameters.

We enforce the following constraints to ensure symmetry, continuity, smoothness, flat-field response, and interpolation and to reduce the number of parameters. For symmetry of the axes

$$
\forall(x, y), f(x, y)=f(y, x)
$$

For continuity between pieces

$$
\begin{aligned}
\forall x, \lim _{y \rightarrow 1-} f(x, y) & =\lim _{y \rightarrow 1+}(x, y) \\
\forall y, \lim _{x \rightarrow 1-} f(x, y) & =\lim _{x \rightarrow 1+} f(x, y) \\
\forall x, f(x, 2) & =0 \\
\forall y, f(2, y) & =0 .
\end{aligned}
$$

For a continuous first-derivative between pieces

$$
\begin{aligned}
\forall x,\left.\frac{\partial f}{\partial y}\right|_{(x, 0)} & =0 \\
\forall y,\left.\frac{\partial f}{\partial x}\right|_{(0, y)} & =0 \\
\forall x,\left.\lim _{y \rightarrow 1-} \frac{\partial f}{\partial y}\right|_{(x, y)} & =\lim _{y \rightarrow 1+1+\left.\frac{\partial f}{\partial y}\right|_{(x, y)}} \\
\forall y,\left.\lim _{x \rightarrow 1} \frac{\partial f}{\partial x}\right|_{(x, y)} & =\left.\lim _{x \rightarrow 1+} \frac{\partial f}{\partial x}\right|_{(x, y)} \\
\forall x,\left.\frac{\partial f}{\partial y}\right|_{(x, 2)} & =0 \\
\forall y,\left.\frac{\partial f}{\partial x}\right|_{(2, y)} & =0 .
\end{aligned}
$$

For a flat-field response and interpolation

$$
\begin{aligned}
\forall(x, y), \sum_{m=-\infty}^{\infty} \sum_{n=-\infty}^{\infty} f(x-m, y-n) & =1 \\
\forall x, f(x, 1) & =0 \\
\forall y, f(1, y) & =0 .
\end{aligned}
$$

Equations (30) and (31) actually constrain the solution more than is necessary for 2-D interpolation, but taken with the other constraints, reduce the number of free parameters from 112 to 2 . 


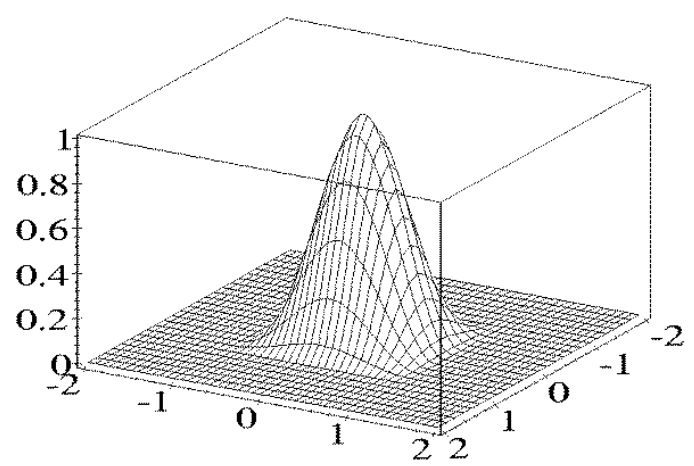

A. $f_{0}(x) f_{0}(y)$

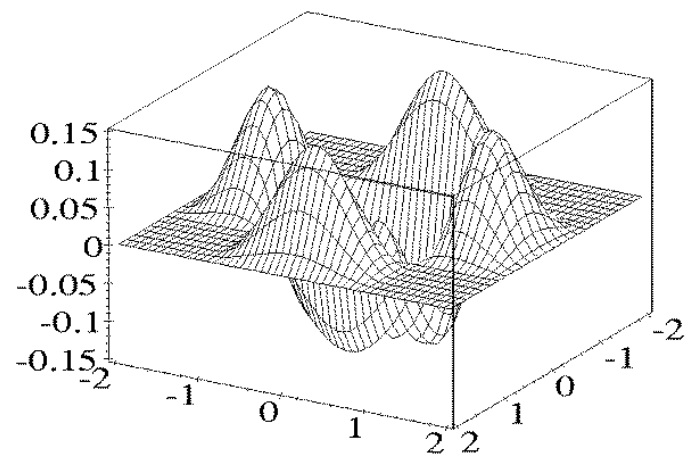

B. $f_{0}(x) f_{1}(y)+f_{1}(x) f_{0}(y)$

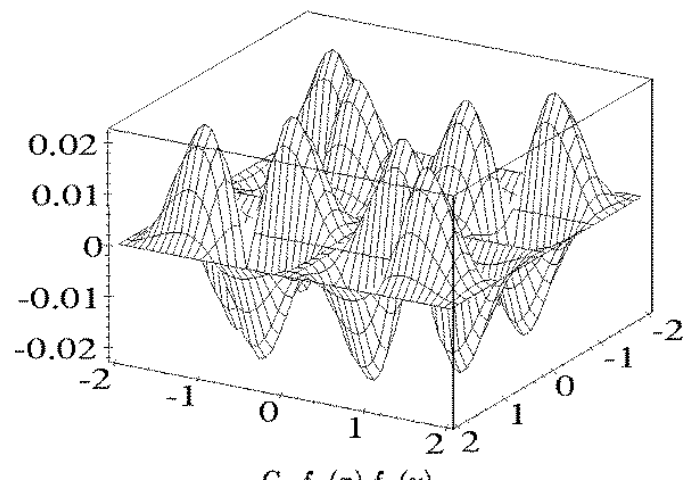

C. $f_{1}(x) f_{1}(y)$

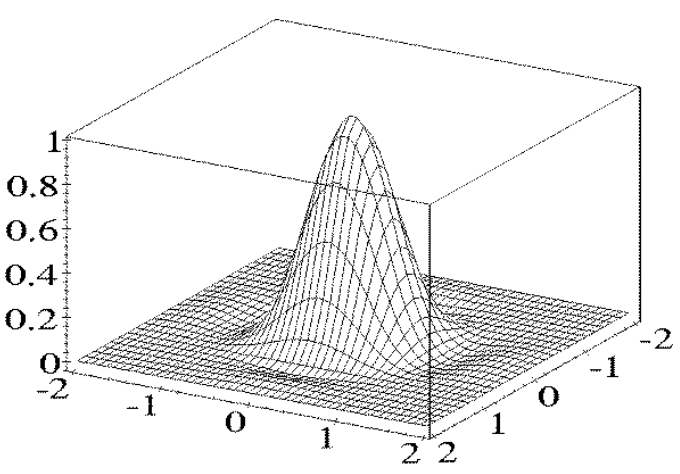

D. $\hat{f}_{0}(u) \hat{f}_{0}(v)$

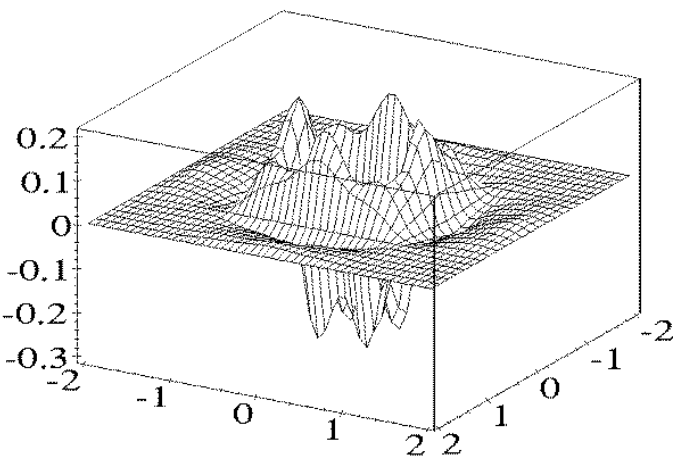

E. $\hat{f}_{0}(x) \hat{f}_{1}(y)+\hat{f}_{1}(x) \hat{f}_{0}(y)$

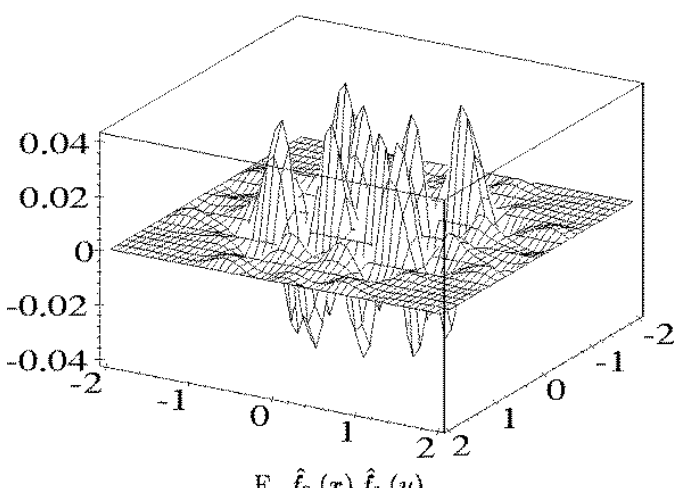

F. $\hat{f}_{0}(x) \hat{f}_{1}(y)$

Fig. 1. Two-dimensional PCC components (spatial-domain left and Fourier-domain right).

With these constraints, the 2-D PCC kernel can be written as the sum of the traditional, separable kernel $f_{s}$ in (16) and an additional term weighted by a new parameter $\beta$

$$
f(x, y)=f_{s}(x, y)+\beta f_{n}(x, y)
$$

where

$$
f_{n}(x, y)=f_{1}(x) f_{1}(y)
$$

with the kernel component $f_{1}$ defined in (13). The new term in the 2-D filter is the separable product of a component of the traditional 1-D derivation. The details of this derivation are provided in Appendix A. Expanding (32) yields

$$
\begin{aligned}
f(x, y)= & \left(f_{0}(x)+\alpha f_{1}(x)\right)\left(f_{0}(y)+\alpha f_{1}(y)\right) \\
& +\beta f_{1}(x) f_{1}(y) \\
\hat{f}(u, v)= & \left(\hat{f}_{0}(u)+\alpha \hat{f}_{1}(u)\right)\left(\hat{f}_{0}(v)+\alpha \hat{f}_{1}(v)\right) \\
& +\beta \hat{f}_{1}(u) \hat{f}_{1}(v) .
\end{aligned}
$$

With $\beta=0$, (32) is identical to the traditional separable PCC kernel.

The three components of the kernel are illustrated in Fig. 1(a)-1(c) and the three corresponding Fourier transforms are illustrated in Fig. 1(d)-1(f).

\section{ANALYSES}

\section{A. Fidelity and Mean-Square-Error}

This section describes how the values of the parameters $\alpha$ and $\beta$ in (34) (and (35)) can be set to maximize the fidelity [5] of the output. Fidelity can be analyzed in the Fourier domain using a simple system model with sampling and interpolation. For image $p$, formed by sampling a scene $s$

$$
p(x, y)=s(x, y) \Perp(x, y)
$$


where $\Perp$ is a uniform lattice with Dirac-delta sampling impulses $\delta$

$$
\Perp(x, y)=\sum_{m=-\infty}^{\infty} \sum_{n=-\infty}^{\infty} \delta(x-m, y-n)
$$

the corresponding Fourier-domain equation is

$$
\hat{p}(u, v)=\sum_{\mu=-\infty}^{\infty} \sum_{\nu=-\infty}^{\infty} \hat{s}(u-\mu, v-\nu) .
$$

Spatial sampling causes a folding or aliasing of components in the Fourier domain spectrum as shown in (38).

The fidelity of the interpolated image $r$ formed by spatial convolution of the image $p$ with the kernel $f$ [as in (1)]

$$
\begin{aligned}
& r(x, y)=\sum_{m=-\infty}^{\infty} \sum_{n=-\infty}^{\infty} p[m, n] f(x-m, y-n) \\
& \hat{r}(u, v)=\hat{p}(u, v) \hat{f}(u, v)
\end{aligned}
$$

is determined by the expected mean-square error (MSE) $\epsilon^{2}$ after sampling and interpolation

$$
\begin{aligned}
\epsilon^{2} & =\mathcal{E}\left\{\int_{-\infty}^{\infty} \int_{-\infty}^{\infty}|r(x, y)-s(x, y)|^{2} d x d y\right\} \\
& =\mathcal{E}\left\{\int_{-\infty}^{\infty} \int_{-\infty}^{\infty}|\hat{r}(u, v)-\hat{s}(u, v)|^{2} d u d v\right\} .
\end{aligned}
$$

If the aliased components are uncorrelated [6], then the expected MSE can be expressed in terms of the scene power spectrum and filter transfer function

$$
\begin{aligned}
\epsilon^{2}= & \int_{-\infty}^{\infty} \int_{-\infty}^{\infty} \hat{\Phi}_{s}(u, v)-2 \hat{f}(u, v) \hat{\Phi}_{s}(u, v) \\
& +|\hat{f}(u, v)|^{2} \sum_{\mu=-\infty}^{\infty} \sum_{\nu=-\infty}^{\infty} \hat{\Phi}_{s}(u-\mu, v-\nu) d u d v
\end{aligned}
$$

where $\hat{\Phi}_{s}$ is the scene power-spectrum (the expected power as a function of frequency)

$$
\hat{\Phi}_{s}(u, v)=\mathcal{E}\left\{|\hat{s}(u, v)|^{2}\right\} .
$$

The kernel parameter $\beta$ that minimizes expected MSE in (42) (and so maximizes fidelity) can be determined by substituting the expression for the transfer function of 2-D PCC kernel from (35) into (42) and solving for $\beta$ where the partial derivative of $\epsilon^{2}$ with respect to $\beta$ is equal to zero

$$
\frac{\partial \epsilon^{2}}{\partial \beta}=0
$$

The value of $\beta$ that yields optimal fidelity is (see (45) at the bottom of the page). Note that the optimal value for $\beta$ in (45) is a function of the scene power-spectrum and the kernel components with parameter $\alpha$.
The optimal value for the kernel parameter $\alpha$ can be determined by numerical analysis. That is, for a given powerspectrum, the value of $\alpha$ can be varied to determine the value that minimizes the expected MSE in (42).

A similar analysis can be used to derive the least-squares optimal reconstruction function, known as the Wiener filter [6]

$$
\hat{f}_{w}(u, v)=\frac{\hat{\Phi}_{s}(u, v)}{\sum_{\mu=-\infty}^{\infty} \sum_{\nu=-\infty}^{\infty} \hat{\Phi}_{s}(u-\mu, v-\nu)} .
$$

From (42), the expected mean-square error for the Wiener filter is

$$
\epsilon_{w}^{2}=\int_{-\infty}^{\infty} \int_{-\infty}^{\infty} \hat{\Phi}_{s}(u, v)-\hat{\Phi}_{s}(u, v) \hat{f}_{w}(u, v) d u d v
$$

The Wiener filter is an approximator, not an interpolator, and its unconstrained spatial support makes it relatively impractical, but all other filters have greater expected mean-square error. The mean-square error for any filter exceeds that of the Wiener filter by its difference with the Wiener filter weighted by the scene power-spectrum:

$$
\epsilon_{f}^{2}=\epsilon_{w}^{2}+\int_{-\infty}^{\infty} \int_{-\infty}^{\infty} \hat{\Phi}_{s}(u, v)\left|\hat{f}_{w}(u, v)-\hat{f}(u, v)\right|^{2} d u d v
$$

\section{B. Scene Models and Optimal Parameters}

This section considers three simple scene models—a circular pulse, a rotated square, and a Markov random field—and illustrates the optimal parameters for each model.

Consider a scene that contains a circular pulse. Such a scene contains an edge point for all orientations. The scene powerspectrum is a function of the diameter $d$

$$
\hat{\Phi}_{s}(u, v)=\left|\frac{d}{r} J_{1}(2 \pi d r)\right|^{2}
$$

where $r=\sqrt{u^{2}+v^{2}}$ and $J_{1}$ is the first-order Bessel function. The optimal PCC parameters $\alpha$ and $\beta$ can be determined for values of the diameter $d$ as described in Section III-A. In the results presented here, (42) is evaluated numerically using a frequency space of -16 to 16 cycles/pixel in a $512 \times 512$ coefficient array (yielding $16 \times 16$ pixel space).

Fig. 2 graphs the optimal parameters for 2-D PCC, given a scene with a circular pulse, as a function of the diameter. For a circular pulse with diameter $d=2$ pixels, the optimal values for 2-D PCC are about $\alpha=-0.29$ and $\beta=0.05$. The optimal parameters for circular pulses with diameter larger than 2 pixels vary slowly with diameter. For comparison, a separable analysis for this model indicates an optimal value of $\alpha$ between -0.45 and -0.50 for larger diameters $d>1.25$ pixels.

$$
\beta=\frac{\int_{-\infty}^{\infty} \int_{-\infty}^{\infty}\left(\hat{\Phi}_{s}(u, v)-\sum_{\mu=-\infty}^{\infty} \sum_{\nu=-\infty}^{\infty} \hat{\Phi}_{s}(u-\mu, v-\nu) \hat{f}_{s}(u, v)\right) \hat{f}_{n}(u, v) d u d v}{\int_{-\infty}^{\infty} \int_{-\infty}^{\infty} \sum_{\mu=-\infty}^{\infty} \sum_{\nu=-\infty}^{\infty} \hat{\Phi}_{s}(u-\mu, v-\nu)\left(\hat{f}_{n}(u, v)\right)^{2} d u d v}
$$




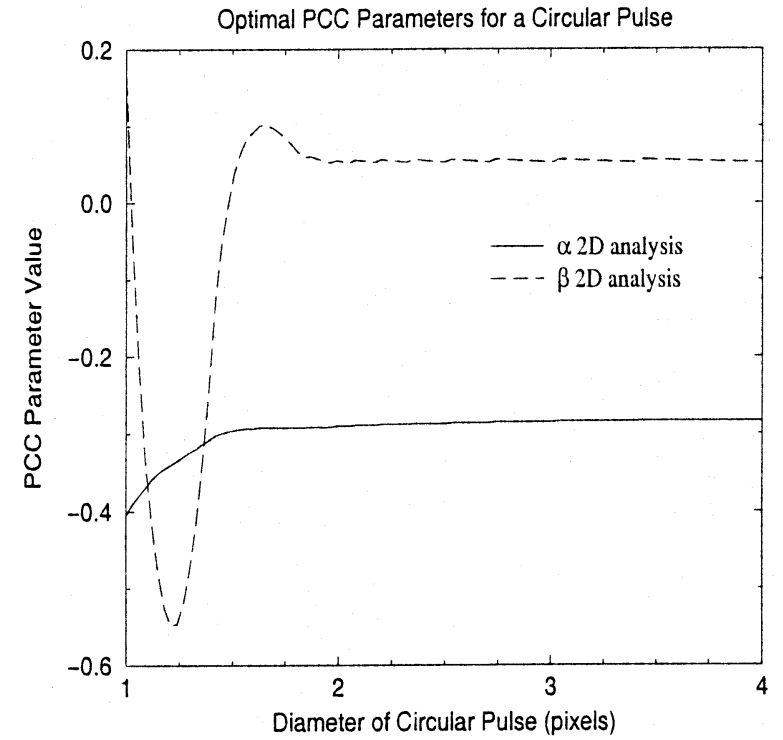

Fig. 2. Optimal PCC parameters for a circular pulse as a function of pulse diameter.

Consider a scene that contains a square pulse of dimension $s$ and rotated by angle $\theta$. The scene power-spectrum is

$$
\hat{\Phi}_{s}(u, v)=\left|\operatorname{sinc}\left(s u^{\prime}\right) \operatorname{sinc}\left(s v^{\prime}\right)\right|^{2}
$$

where $u^{\prime}=u \cos (\theta)+v \sin (\theta)$ and $v^{\prime}=-u \sin (\theta)+v \cos (\theta)$. The optimal PCC parameters $\alpha$ and $\beta$ can be determined for values of $s$ and $\theta$.

Fig. 3 graphs the optimal parameters for 2-D PCC, given a scene with a square pulse with dimension $s=2$ pixels, as a function of the rotation angle. The figure illustrates that the optimal parameters are different depending on the angle of rotation. The optimal value for the parameter $\alpha$ in 2-D PCC ranges from -0.08 for rotation 0 down to -0.39 for rotation $\pi / 4$. For comparison, a separable analysis for this model indicates an optimal value of between -0.16 for rotation 0 down to -0.68 for rotation $\pi / 4$.

Consider a more complex and general scene model-a 2-D, isotropic, Markov random field with mean-spatial-detail $d$. This power-spectrum is appropriate for modeling both images and geostatical quantities [7]

$$
\hat{\Phi}_{s}(u, v)=\frac{2 \pi d^{2}}{\left(1+4 \pi^{2} d^{2}\left(u^{2}+v^{2}\right)\right)^{\frac{3}{2}}} .
$$

The mean-spatial-detail can be interpreted as the average size of elements in the scene, i.e., scenes with larger objects (relative to the sampling interval) have larger mean-spatial-detail. The optimal PCC parameters $\alpha$ and $\beta$ can be determined for values of the mean-spatial-detail $d$.

Fig. 4 graphs the optimal parameters for 2-D PCC, given a scene that is a Markov field, as a function of mean-spatial-detail. For mean-spatial-detail $d=1$ pixel, the optimal parameters are $\alpha=0.00$ and $\beta=0.59$. As the mean-spatial-detail $d$ increases, the optimal values of both $\alpha$ and $\beta$ decrease, with the rate of decrease diminishing, to about $\alpha=-0.24$ and $\beta=0.19$ at mean-spatial-detail $d=4$ pixels. The magnitude of the parameter $\beta$ decreases as the mean-spatial-detail increases. This is expected, because as can be seen in Fig. 1(f), the

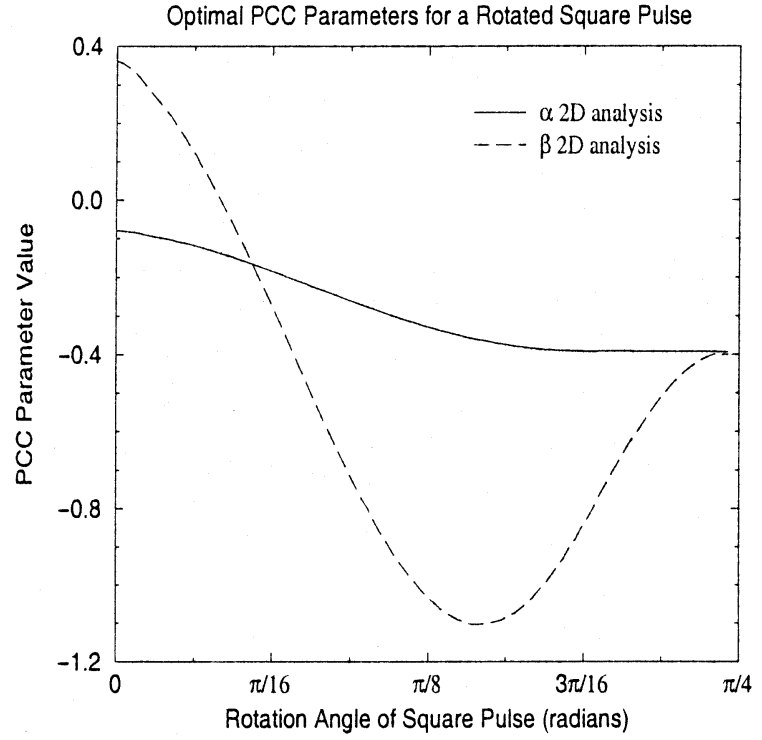

Fig. 3. Optimal PCC parameters for a rotated square pulse (dimension 2 pixels) as a function of rotation angle.

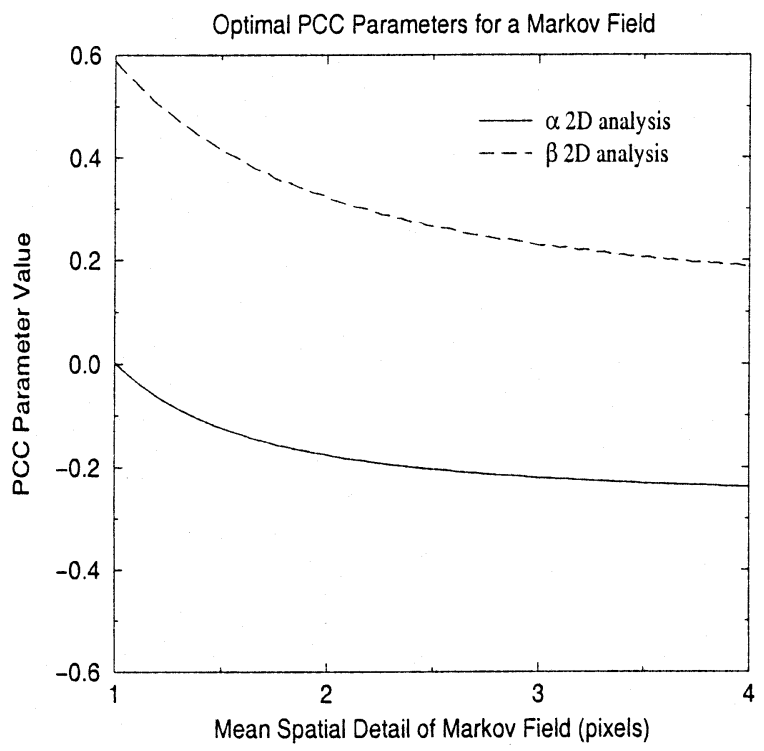

Fig. 4. Optimal kernel parameters for a Markov random field as a function of mean-spatial-detail.

component of the filter controlled by the $\beta$ parameter affects primarily high-frequency signal components. For comparison, a separable analysis for this model indicates an optimal value of -0.22 for mean-spatial-detail $d=1$ pixel and the optimal value decreasing (and rate of decrease diminishing) to -0.44 for mean-spatial-detail $d=4$ pixels.

\section{PERformanCE}

This section examines three issues of performance: optimal fidelity, robustness, and computation. Optimal fidelity concerns how well the system can achieve the goal of interpolating the scene. Robustness concerns how well the system performs when there is a mismatch between the kernel design and the scene (i.e., the kernel is applied to a scene with different characteristics than were assumed in the design). Computation concerns 

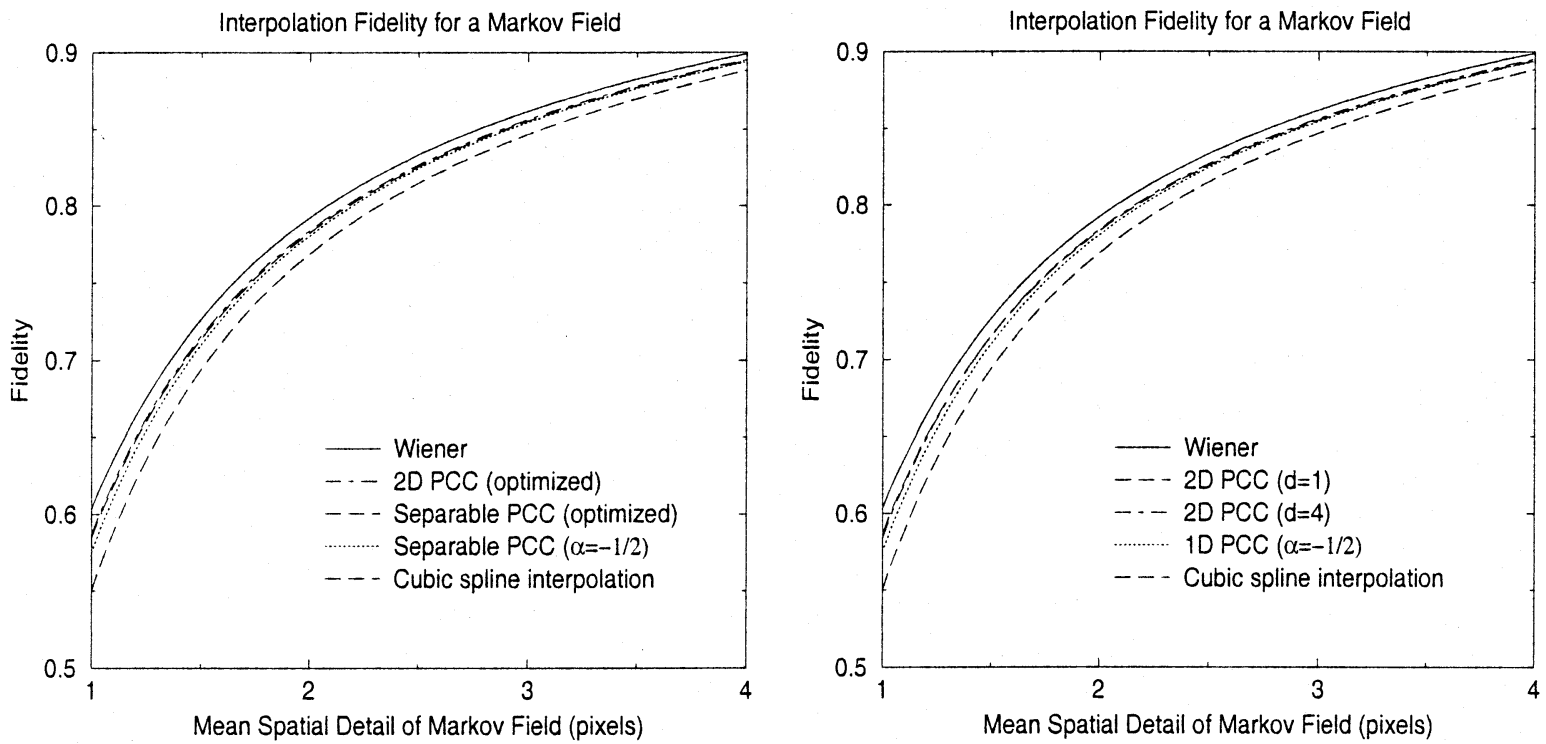

Fig. 5. Interpolation fidelity for a scene with a Markov random field as a function of mean-spatial-detail.
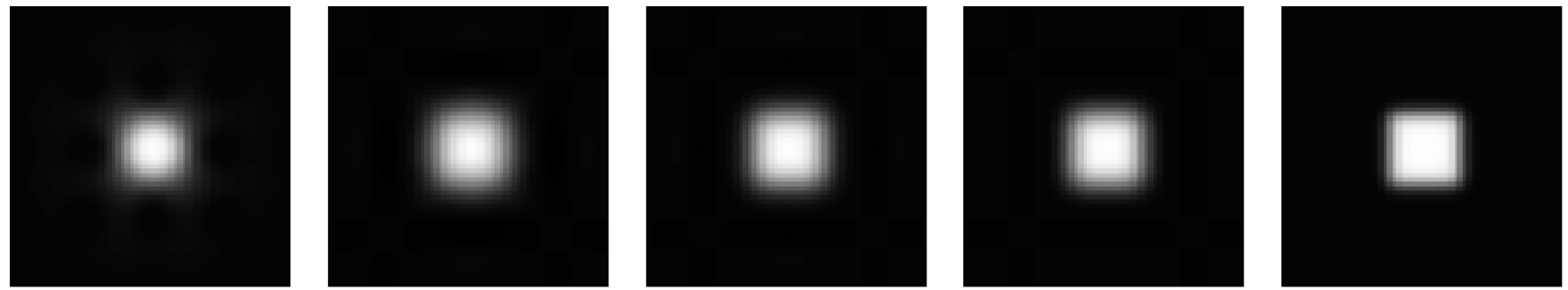

A. Transfer functions, left-to-right: Wiener, optimal 2D PCC, optimal separable PCC, separable PCC $(\alpha=-0.5)$, cubic spline interpolation
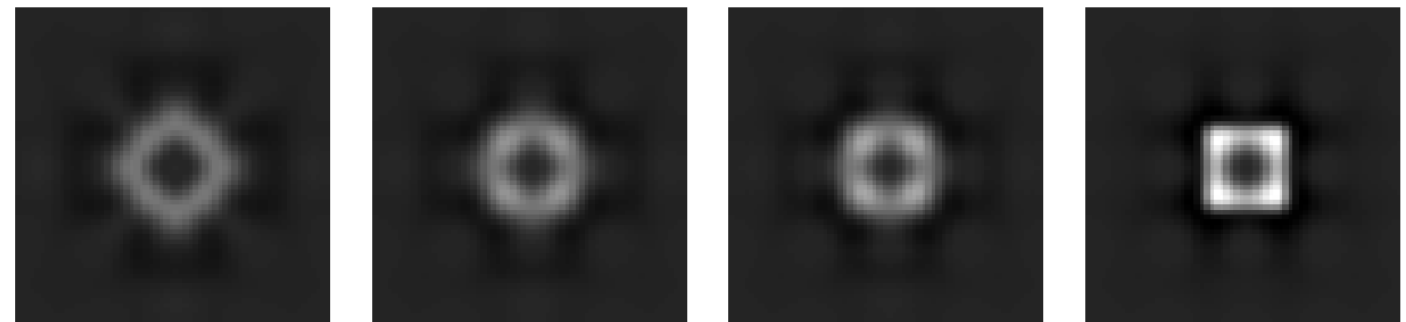

B. Differences with Wiener transfer function, left-to-right: optimal 2D PCC, optimal separable PCC, separable PCC ( $\alpha=-0.5)$, cubic spline interpolation

Fig. 6. Various filter transfer functions (frequencies -2 to 2 cycles/pixel for a Markov field with mean-spatial-detail $d=2$ ) and the differences from the Wiener transfer function.

how many operations and how much memory are required in the processing.

This section employs the 2-D, isotropic, Markov random field scene model described in Section III-B, in which Fig. 4 presents the optimal parameters for PCC as a function of the mean-spatial-detail. The independent variable in the experiments is the mean-spatial-detail, with values $d=1$ to 4 pixels. The reported dependent variables are

1) fidelity for the optimal kernel;

2) fidelity for mismatched kernels (derived for one meanspatial-detail and applied to scenes with another meanspatial-detail).
Fidelity [5] is a normalized measure of image quality as a function of the mean-square error

$$
\mathcal{F}=1-\frac{\epsilon^{2}}{\int_{-\infty}^{\infty} \int_{-\infty}^{\infty} \hat{\Phi}_{s}(u, v) d u d v} .
$$

The greatest fidelity possible is 1.0 , where the mean-square error is 0 .

Fig. 5(a) graphs the fidelity for 2-D PCC with the optimal parameter values and the fidelity for several other filters, including: Wiener, separable PCC with optimal parameters (determined by separable analysis), separable PCC with a fixed parameter $\alpha=-0.5$, and cubic spline interpolation [8]. The 


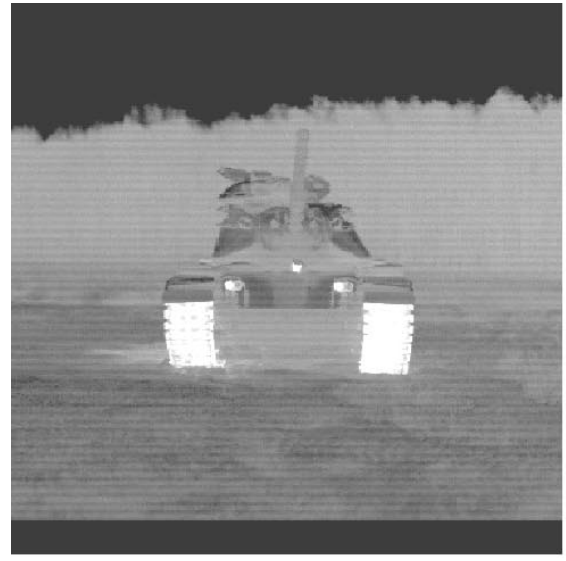

A. Infrared scene

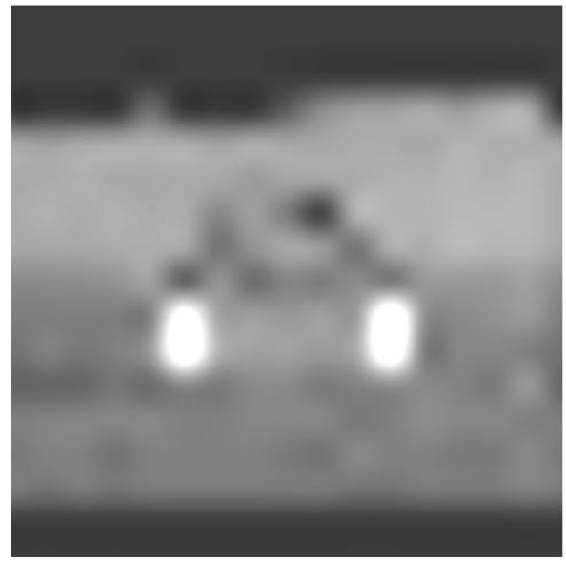

D. Optimal separable PCC

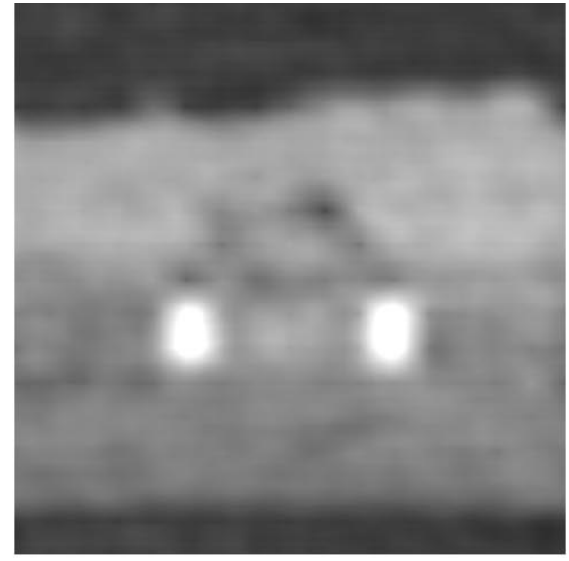

B. Wiener filter

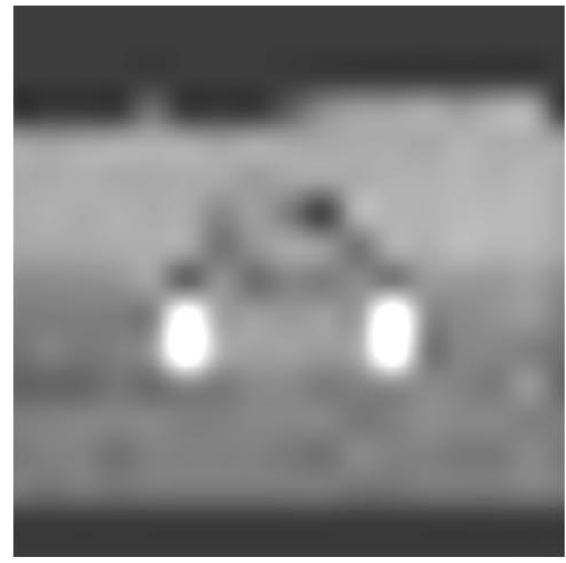

E. Separable PCC $(\alpha=-0.5)$

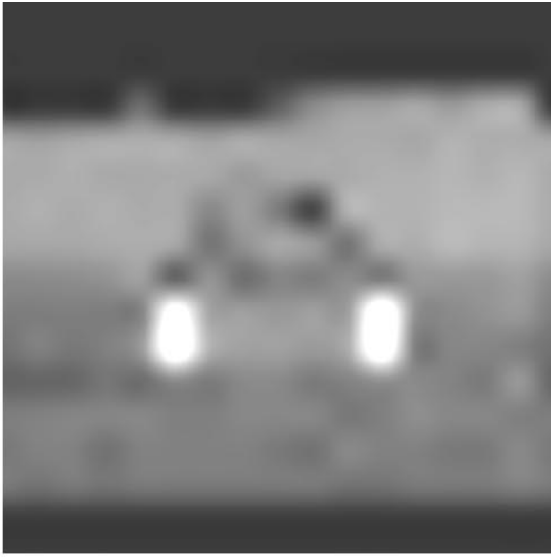

C. Optimal 2D PCC

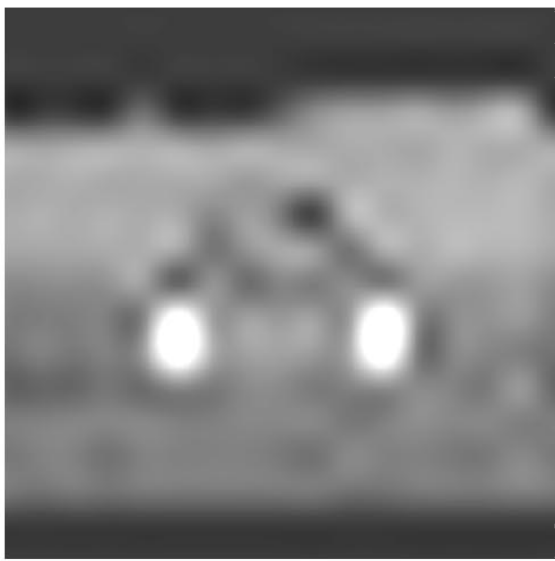

F. Cubic spline interpolation

Fig. 7. Images produced by various methods.

Wiener filter (46) has the best fidelity that can be expected (47). For the Markov field, the 2-D PCC with optimal parameters out-performs all of the other listed methods, but the differences are relatively small. In particular, the improvement of the optimal 2-D PCC over the optimal separable PCC is smallest of the methods compared. Table I summarizes the fidelity values for several levels of mean-spatial-detail.

The reason for the superior performance of the optimal PCC filters in this problem can be understood by examining the filter transfer functions, illustrated in Fig. 6(a) (frequencies -2 to 2 cycles/pixel for a Markov field with mean-spatial-detail $d=2$ pixels). The Wiener filter achieves the best-possible expected result for the signal and aliasing present in this example. The transfer functions of cubic spline interpolation and separable PCC with $\alpha=-0.5$ are much more square than the Wiener transfer function, with the differences illustrated in Fig. 6(b). The transfer functions of the optimal separable PCC and optimal 2-D PCC are closer to the Wiener transfer function, explaining their better performance. This is a single example, but illustrates the type of analysis that can be used to understand performance.

Fig. 5(b) graphs the fidelity for mismatched 2-D PCC. Mismatch means that the 2-D PCC parameters were derived for a scene with a different mean-spatial detail than was actually presented. Here, the 2-D PCC designed for a Markov field with mean-spatial-detail $d=1$ and the 2-D PCC designed for a
TABLE I

Fidelity of SEVERal Processing Methods ApPlied to a SAmPled MARKOV FIELD WITH VARIOUS MEAN-SPATIAL-DETAIL

\begin{tabular}{l|c}
\hline Processing Method & Fidelity \\
\hline Wiener & 0.8515 \\
2D PCC (optimized) & 0.8303 \\
Separable PCC (optimized) & 0.8300 \\
Separable PCC $(\alpha=-0.5)$ & 0.8297 \\
Cubic spline interpolation & 0.8236 \\
\hline
\end{tabular}

Markov field with mean-spatial-detail $d=4$ both are considered for Markov fields with mean-spatial-detail from 1 to 4 . For the Markov field, even with a design mismatch, the 2-D PCC yields better fidelity than separable PCC with $\alpha=-0.5$ and cubic spline interpolation. Table I includes the fidelity for the mismatched PCC.

Cubic convolution is attractive because the output can be computed relatively simply. With the separable kernel, the convolution can be performed as two, 1-D operations. As has been shown, the 2-D PCC kernel is the sum of two separable functions. Therefore, the convolution still can be performed as 1-D operations, with two computations in each dimension. 
TABLE II

Fidelity of Several Processing Methods Applied to a SAmpled INFRARED SCENE

\begin{tabular}{l|r|r|r}
\hline & \multicolumn{3}{|c}{ Mean-Spatial-Detail } \\
Processing Method & $d=1$ & $d=2$ & $d=4$ \\
\hline Wiener & 0.6034 & 0.7935 & 0.8994 \\
2D PCC (optimized) & 0.5867 & 0.7854 & 0.8954 \\
2D PCC $(d=1)$ & 0.5867 & 0.7844 & 0.8945 \\
2D PCC $(d=4)$ & 0.5842 & 0.7852 & 0.8954 \\
Separable PCC (optimized) & 0.5846 & 0.7839 & 0.8947 \\
Separable PCC $(\alpha=-0.5)$ & 0.5747 & 0.7819 & 0.8941 \\
Cubic spline interpolation & 0.5501 & 0.7701 & 0.8885 \\
\hline
\end{tabular}

So, computing output for 2-D PCC requires no more than twice the number of operations as the separable convolution. Two-dimensional PCC is a local operation that requires very little memory.

\section{EXAMPLE IMAGE}

The 2-D PCC is derived based on quantitative optimization of fidelity (subject to constraints). Many image processing applications are quantitative, but some applications are visual, so it is worthwhile to also consider the visual quality produced by 2-D PCC. Fig. 7(a) presents an infrared scene of an M60 tank. This $512 \times 512$ scene is sampled to $16 \times 16$ and then reconstructed to $512 \times 512$ using several filters: Wiener, optimal 2-D PCC, optimal separable PCC, separable PCC with $\alpha=-0.5$, and cubic spline interpolation. The images produced by these methods are presented in Fig. 7. The fidelity for each of the methods for this image are presented in Table II. Two-dimensional PCC achieves the highest fidelity of the methods compared. As with the mathematical expectations, the differences between the methods are small—both visually and quantitatively.

\section{CONCLUSION}

Emerging technologies such as commercial digital still-cameras and high-resolution digital television are increasing interest in 2-D interpolation algorithms designed to balance computational complexity and accuracy.

Piecewise cubic convolution (PCC) is a relative efficient method of interpolation with performance that exceeds nearest-neighbor and bi-linear methods. The traditional PCC kernel is derived in one-dimension and the kernel is generalized to two dimensions by assuming separability. However, most scenes and imaging systems are not separable.

This paper develops a closed-form, 2-D derivation for PCC. Experiments with image models and actual images indicate that 2-D PCC produces high-fidelity images, is robust, and is efficient. However, the improvements over separable PCC are small. Future improvements in 2-D PCC may be realized by relaxing some of the constraints imposed on the derivation. More parameters would both improve performance and provide greater flexibility.

\section{APPENDIX}

\section{Details of THE Two-Dimensional DERIVATION}

The reduction [9] employs two propositions.

Proposition 1: For $P(x, y)$, a polynomial of $x$ and $y$ with degree $n>0$, if $\forall y \in \Re, P\left(x_{0}, y\right)=0$, then $P(x, y)$ must have factor $\left(x-x_{0}\right)$.

Proposition 2: For $Q(x, y)=\left(x-x_{0}\right) P(x, y)$, with $P(x, y)$ a polynomials of $x$ and $y$ with degree $n>0$, if $\forall y \in \Re, \partial Q(x, y) /\left.\partial x\right|_{x=x_{0}}=0$, then $Q(x, y)$ must have factor $\left(x-x_{0}\right)^{2}$.

Then, with the constraints in (19)-(22), (27),(28), and (30),(31), the kernel can be written more simply as:

$$
\begin{aligned}
& f_{a}(x, y)=(x-1)(y-1) \sum_{j=0}^{4} \sum_{k=0}^{4-j} a_{j k} x^{j} y^{k} \\
& f_{b}(x, y)=(x-1)(y-1)(x-2)^{2} \sum_{j=0}^{2} \sum_{k=0}^{2-j} b_{j k} x^{j} y^{k} \\
& f_{c}(x, y)=(x-1)(y-1)(x-2)^{2}(y-2)^{2} c_{00} \\
& f_{d}(x, y)=(x-1)(y-1)(y-2)^{2} \sum_{j=0}^{2} \sum_{k=0}^{2-j} d_{j k} x^{j} y^{k}
\end{aligned}
$$

Note that the coefficients in (53)-(56) are distinct from those in (17).

Proceeding from (53), the smoothness constraint in (23) on $f_{a}$ at $y=0$ requires:

$$
\begin{aligned}
& \forall x,(x-1)\left(a_{40} x^{4}+\left(a_{30}-a_{31}\right) x^{3}\right. \\
& \left.\quad+\left(a_{20}-a_{21}\right) x^{2}+\left(a_{10}-a_{11}\right) x+\left(a_{00}-a_{01}\right)\right)=0
\end{aligned}
$$

Then:

$$
\begin{aligned}
& a_{40}=0 \\
& a_{30}=a_{31} \\
& a_{20}=a_{21} \\
& a_{10}=a_{11} \\
& a_{00}=a_{01} .
\end{aligned}
$$

With symmetric axes:

$$
\begin{aligned}
& a_{40}=a_{04}=0 \\
& a_{30}=a_{31}=a_{03}=a_{13} \\
& a_{20}=a_{21}=a_{12}=a_{02} \\
& a_{10}=a_{11}=a_{01}=a_{00} .
\end{aligned}
$$

Next, the smoothness constraint in (23) on $f_{b}$ at $y=0$ requires:

$$
\forall x,(x-1)(x-2)^{2}\left(b_{20} x^{2}+\left(b_{10}-b_{11}\right) x+\left(b_{00}-b_{01}\right)\right)=0 .
$$

So:

$$
\begin{aligned}
& b_{20}=0 \\
& b_{10}=b_{11} \\
& b_{00}=b_{01} .
\end{aligned}
$$


With symmetric axes

$$
\begin{aligned}
& b_{20}=d_{02}=0 \\
& b_{10}=b_{11}=d_{01}=d_{11} \\
& b_{00}=b_{01}=d_{00}=d_{10} .
\end{aligned}
$$

Next, after simplification using the equalities in (60), the smoothness constraint in (26) on $f_{c}$ and $f_{d}$ at $x=1$ requires

$$
\forall y,(y-1)(y-2)^{2}\left(-2 d_{11} y+c_{00}-2 d_{10}-d_{20}\right)=0 .
$$

Then:

$$
\begin{aligned}
& d_{11}=0 \\
& c_{00}=2 d_{10}+d_{20} .
\end{aligned}
$$

Next, after simplification using the equalities in (58), (60), and (62), the smoothness constraint in (26) on $f_{a}$ and $f_{b}$ at $x=1$ requires

$$
\begin{gathered}
\forall y,(y-1)\left(2 a_{13} y^{3}+\left(2 a_{02}+a_{22}-b_{02}\right) y^{2}+\right. \\
\left(2 a_{00}+a_{02}+a_{13}-b_{01}\right) y+ \\
\left.2 a_{00}+a_{02}+a_{13}-b_{01}\right)=0 .
\end{gathered}
$$

Then

$$
\begin{aligned}
& a_{13}=0 \\
& b_{02}=a_{22}+2 a_{02} \\
& b_{01}=2 a_{00}+a_{02} .
\end{aligned}
$$

With symmetric axes

$$
\begin{aligned}
& b_{02}=d_{20}=a_{22}+2 a_{02} \\
& b_{01}=d_{10}=2 a_{00}+a_{02} .
\end{aligned}
$$

Finally, observing that from the constraints related to flatfield response and interpolation

$$
a_{00}=1
$$

leaves only two free parameters in defining the kernel, $a_{22}$ and $a_{02}$.

The function in the two free parameters is

$$
\begin{aligned}
f_{a}(x, y)= & (x-1)(y-1) \\
& \times\left(x y+x+y+1+a_{02}\left(x^{2} y+x y^{2}+x^{2}+y^{2}\right)\right. \\
& \left.\quad+a_{22} x^{2} y^{2}\right) \\
f_{b}(x, y)= & (x-1)(y-1)(x-2)^{2} \\
& \times\left(\left(a_{02}+2\right)(y+1)+\left(2 a_{02}+a_{22}\right) y^{2}\right) \\
f_{c}(x, y)= & (x-1)(y-1)(x-2)^{2} \\
& \times(y-2)^{2}\left(4 a_{02}+a_{22}+4\right) \\
f_{d}(x, y)= & (x-1)(y-1)(y-2)^{2} \\
& \times\left(\left(a_{02}+2\right)(x+1)+\left(2 a_{02}+a_{22}\right) x^{2}\right)
\end{aligned}
$$

This formulation can be written using the same slope parameter used in the 1-D parametric formulation

$$
\alpha=\left.\frac{\partial f}{\partial x}\right|_{(1,0)}=\left.\frac{\partial f}{\partial y}\right|_{(0,1)}
$$

with $a_{02}=-(\alpha+2)$ and $a_{22}=\beta+(\alpha+2)^{2}$. The formulation with $\alpha$ and $\beta$ is shown in (32).

\section{REFERENCES}

[1] S. S. Rifman, "Digital rectification of ERTS multispectral imagery," in Proc. Symp. Significant Results Obtained From BRTS-1, vol. I, sec. B, 1973, NASA SP-327, pp. 1131-1142.

[2] S. K. Park and R. A. Schowengerdt, "Image reconstruction by parametric cubic convolution," Comput. Vis., Graph., Image Process., vol. 23, pp. $258-272,1983$.

[3] F. C. Billingsley, "Data preprocessing and processing," in Manual of Remote Sensing, 2nd ed, R. N. Colwell, Ed. Falls Church, VA: Amer. Soc. Photogramm., 1983, pp. 258-272.

[4] R. G. Keys, "Cubic convolution interpolation for digital image processing," IEEE Trans. Acoust., Speech, Signal Processing, vol. ASSP-29, no. 6, pp. 1153-1160, 1981.

[5] E. H. Linfoot, "Transmission factors and optical design," J. Opt. Soc. Amer., vol. 46, no. 9, pp. 740-752, 1956.

[6] C. L. Fales, F. O. Huck, J. A. McCormick, and S. K. Park, "Wiener restoration of sampled image data: end-to-end analysis," J. Opt. Soc. Amer. A, vol. 5, no. 3, pp. 300-314, 1988.

[7] R. A. Schowengerdt, Remote Sensing: Models and Methods for Image Processing, 2nd ed. Orlando, FL: Academic, 1997.

[8] M. Unser, A. Aldroubi, and M. Eden, "Fast B-spline transforms for continuous image representation and interpolation," IEEE Trans. Pattern Anal. Machine Intell., vol. 13, no. 3, pp. 277-285, 1991.

[9] F. Geng, "Image Reconstruction with Two-Dimensional Piecewise Polynomial Convolution," M.S. thesis, Comput. Sci. Eng. Dept., Univ. Nebraska—Lincoln, 1998.

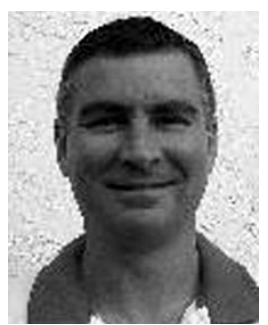

Stephen E. Reichenbach (S'85-M'89) received the Ph.D. degree in computer science from the College of William and Mary, Williamsburg, VA, the M.S. degree in computer science from Washington University, St. Louis, MO, and the B.S. degree from the University of Nebraska-Lincoln (UNL).

$\mathrm{He}$ is a Professor in the Computer Science and Engineering (CSE) Department, UNL. He held a National Research Council research associateship in the Visual Information Processing Laboratory at the NASA Langley Research Center and an ASEE Research Fellowship in the Landsat 7 Project Science Office. From 1996 to 2000, he served as UNL CSE Department Chair. He has authored more than 70 papers on digital image processing and image information systems.

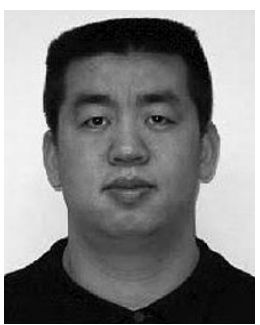

Frank Geng (M'01) received the B.S. degree in computer science from Northwestern Polytechnic University, Xi'An, China, in 1995, and the M.S. degree in computer science from the University of Nebraska-Lincoln in 1998.

He is currently working as an IT Consultant. 